\title{
IMPACT OF NEUTROPHIL FUNCTION ON OUTCOMES OF COMMUNITY-ACQUIRED PNEUMONIA IN PATIENTS WITH CANCER
}

Stefano Aliberti, MD*\#, Asad Amir, MD*, Paula Peyrani, MD\#, John A. Myers, PhD“, Maria Cirino, MD+, Matteo Saporiti, MD*, Francesco Blasi, MD, PhD* and Julio A. Ramirez, MD*

Young Researcher Award: Respiratory Infection Aetiological Diagnosis, sponsored by Brahms

*Institute of Respiratory Disease, University of Milan, Ospedale Maggiore Fondazione IRCCS Policlinico, Mangiagalli e Regina Elena, Via F Sforza 35, Milan 20122, Italy

\#Division of Infectious Diseases, Department of Medicine, University of Louisville, Louisville, Kentucky, USA

'Department of Bioinformatics and Biostatistics, School of Public Health and Information Sciences, University of Louisville, Louisville,

Kentucky, USA

+Division of Pulmonary and Critical Care Medicine, University of Louisville, Louisville, Kentucky, USA

WINNING ABSTRACT: Some literature suggests that outcomes of CAP in patients with solid tumor without neutropenia may be better than in cancer patients with neutropenia. In order to investigate the role of neutrophils in this population, we retrospectively analyzed consecutive patients with CAP admitted to 3 tertiary care hospitals from 01/2001 through 12/2005. HIV patients were excluded. A total of 993 patients were classified as follows: Group 1: no active cancer; Group 2: solid cancer without neutropenia; Group 3: solid cancer without neutropenia or hematologic malignancy. Age, Pneumonia Severity Index (PSI), overall mortality, time to reach clinical stability (TCS) and length of stay in hospital (LOS) are summarized in the table. No significant differences were found for any of the outcomes between group 2 and 3 . Our results suggest that physicians should aggressively manage cancer patients with CAP, regardless of the neutrophil count.

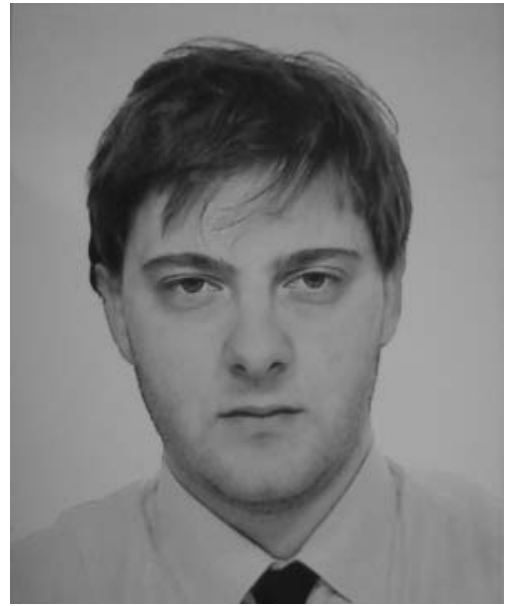

Stefano Aliberti

Institute of Respiratory Disease, University of Milan, Ospedale Maggiore Fondazione IRCCS Policlinico, Milan, Italy

\section{MY JOB AND THE UNIT IN WHICH I WORK}

I work as a visiting research fellow at the Division of Infectious Diseases, University of Louisville (Louisville, KY, USA). Under the leadership of Dr J. Ramirez, the Community-Acquired Pneumonia Organization (CAPO) project was developed and is carried out at this institution. My winning abstract is on a study I performed on the CAPO database.
CAPO is an open organisation, with participation from wellestablished international investigators as well as new investigators with the common goal of fostering international research in the field of community-acquired pneumonia (CAP) [1]. The core study has a retrospective, observational design and a system of internet-based clinical research. Data from randomly selected hospitalised adult patients with a diagnosis of CAP are collected by different institutions around the world using a paper data collection form. Data are then entered into a worldwide web-based data collection system and transferred electronically to the CAPO coordinating centre at the University of Louisville. At the coordinating centre, after discrepancies and inconsistencies in the data are resolved and clarified with the international investigators, cases are submitted to the database. The Division of Infectious Diseases coordinates research, performs secondary data analysis,

TABLE 1 Retrospective analysis of patients from three tertiary care hospitals

\begin{tabular}{lccc} 
& $\begin{array}{c}\text { Group 1 } \\
\mathbf{n}=\mathbf{8 9 6}\end{array}$ & $\begin{array}{c}\text { Group 2 } \\
\mathbf{n}=\mathbf{6 5}\end{array}$ & $\begin{array}{c}\text { Group 3 } \\
\mathbf{n}=\mathbf{3 2}\end{array}$ \\
\hline Age yrs & $64 \pm 16$ & $67 \pm 12$ & $65 \pm 17$ \\
PSI scores & $82 \pm 42$ & $127 \pm 29$ & $122 \pm 26$ \\
TCS days & $3.9 \pm 2.6$ & $4.2 \pm 2.6$ & $4.7 \pm 2.7$ \\
LOS days & $7.4 \pm 7.8$ & $7.7 \pm 8.6$ & $9.0 \pm 8.1$ \\
Mortality & $44(4.9)^{\#}$ & $10(15)^{\#}$ & $3(9.4)$ \\
\hline
\end{tabular}

Data are presented as mean \pm SD or $n$ (\%). PSI: Pneumonia Severity Index; TCS time to reach clinical stability; LOS: length of stay in hospital. ${ }^{*}: p=0.002$ 
coordinates ancillary studies, and supports the preparation of abstracts for presentation and manuscripts for publication. A description of the study protocol, study manual and data collection form is available from the CAPO website [2].

The core clinical study is entitled "An international observational study to evaluate the current management of hospitalized patients with community-acquired pneumonia". The primary objective of the core study is to evaluate the quality of care delivered to hospitalised patients with CAP. Unlike CAP clinical trials, participants in the CAPO observational cohort study include patients with more complex medical problems. The CAPO cohort, therefore, more closely reflects the real-world experience of hospitalised patients with CAP. There are currently $>3,500$ cases of hospitalised patients with CAP enrolled in the CAPO core study, representing 46 institutions from 16 countries.

\section{MY WINNING POSTER AS PART OF MY RESEARCH}

I am a clinical pulmonary fellow at the Institute of Respiratory Diseases at the University of Milan (Milan, Italy), working under the instruction of Prof. Blasi in the field of respiratory infections. Our institute became part of the CAPO project in 2001. Before arriving in Louisville, I worked in a respiratory intensive care unit treating severe pneumonia cases complicated by acute respiratory failure. Among the most difficult to treat and those most likely to have poor outcomes were immunocompromised cancer patients, particularly those with haematological malignancy. In fact, in patients whose immunosuppression is due to cancer, pneumonia accounts for a mortality rate that is higher in comparison to patients with pneumonia and other immunocompromised states [3, 4]. I arrived in Louisville in October 2006 to work on the CAPO project and began investigating the outcomes of cancer patients with CAP using the CAPO database. The strength of the CAPO database arises from the enormous number of patients enrolled and the presence of different clinical outcomes, including not only the in-hospital and CAPrelated mortality, but also the hospital length of stay and the time to clinical stability. Using these data, I was able to conduct analyses of CAP in cancer and neutropenic patients, focusing not only on microorganisms but also on clinical outcomes. My study received international support as investigators were willing to collect additional data for patients previously enrolled in the project in order to strengthen certain variables for analysis in the CAPO database. I performed an "ancillary study," which is a type of study based on the modification of the core cohort-study protocol in which new data are collected. Thanks to the wellestablished web-based system and the strong relationships that exist between CAPO investigators, I was able to perform and conclude this study.

\section{MY RESEARCH AS PART OF MY WORKING GROUP/ RESEARCH TEAM}

The research conducted at the Division of Infectious Diseases primarily focuses on the area of pneumonia. In addition to CAPO, Dr Ramirez launched a nosocomial pneumonia study in order to assess outcomes and improve processes of care. In recent months, concentration on methicillin-resistant Staphylococcus aureus (MRSA) pneumonia has increased in this study, which is strengthened by the retention of the respiratory isolates by the Infectious Diseases Laboratory (Louisville) for genetic analysis. The division also participates in a clinical trial evaluating the efficacy of two Food and Drug Agency approved antibiotics in the treatment of MRSA pneumonia. Besides pneumonia, another new area of interest for this division is in soft tissue infections and osteomyelitis, for which efforts to create a similar database infrastructure are in progress.

Under the direction of Dr Ramirez, the Division of Infectious Disease has created a unique fellowship training programme with a strong combination of patient care and clinical research. All observational and intervention studies are managed by the Director of Clinical Research, Paula Peyrani, MD. The project managers involved in areas such as regulatory compliance and training, logistical management and data validation Ginny Sciortino, CCRC, and Mary Elizabeth Allen, MBA, who is a doctoral student at the School of Public Health (Louisville). Two clinical research fellows, Asad Amir, MD, and Mehdi Mirsaeidi, MD, MPH, are currently working with me on CAPO and other projects. Also involved in research are three clinical fellows and three faculty members, Raul Nakamatsu, MD, Forest Arnold, DO, and Marty Allen, MD. This multidisciplinary team also includes an associate professor with the Kornhauser Health Sciences Library (Louisville), Elizabeth Smigielski, and three part-time statisticians from the School of Public Health who provide statistical support for the CAPO core study, as well as for all other research projects.

\section{THE IMPACT OF MY WORK ON CLINICAL OR RESEARCH PRACTICE}

In analysing the impact of cancer on outcomes of hospitalised patients with community-acquired pneumonia, we surprisingly found that patients without neutropenia had outcomes similar to those with neutropenia. Our findings are consistent with recent literature that has questioned the role of neutropenia in outcomes of patients with both haematological and solid tumours with different infections [5-7]. A possible explanation could be found in the simultaneous impairment of multiple aspects of the host defence system, beside neutropenia, that affects cancer patients hospitalised for communityacquired pneumonia. Our results can impact strongly on clinical practice, suggesting that physicians should manage cancer patients with community-acquired pneumonia aggressively, regardless of the type of cancer or the neutrophil count on admission. Particularly, the lack of neutropenia during the initial evaluation of a cancer patient with community-acquired pneumonia should not be considered an indicator of better clinical outcome.

\section{REFERENCES}

1 Ramirez JA. Fostering international multicenter collaborative research: the CAPO Project. Int J Tuberc Lung Dis 2007; 11: 1062-1065.

2 CAPO. Community-Acquired Pneumonia Organization. www.caposite.com

3 Hilbert G, Gruson D, Vargas F, et al. Noninvasive ventilation in immunosuppressed patients with pulmonary infiltrates, 
fever, and acute respiratory failure. N Engl J Med 2001; 344 481-487.

4 Rano A, Agusti C, Benito N, et al. Prognostic factors of nonHIV immunocompromised patients with pulmonary infiltrates. Chest 2002; 122: 253-261.

5 Kumashi P, Girgawy E, Tarrand JJ, Rolston KV, Raad II, Safdar A. Streptococcus pneumoniae bacteremia in patients with cancer: disease characteristics and outcomes in the era of escalating drug resistance (1998-2002). Medicine (Baltimore) 2005; 84: 303-312.

6 Azoulay E, Moreau D, Alberti C, et al. Predictors of shortterm mortality in critically ill patients with solid malignancies. Intensive Care Med 2000; 26: 1817-1823.

7 Staudinger T, Stoiser B, Mullner M, et al. Outcome and prognostic factors in critically ill cancer patients admitted to the intensive care unit. Crit Care Med 2000; 28: 1322-1328. 